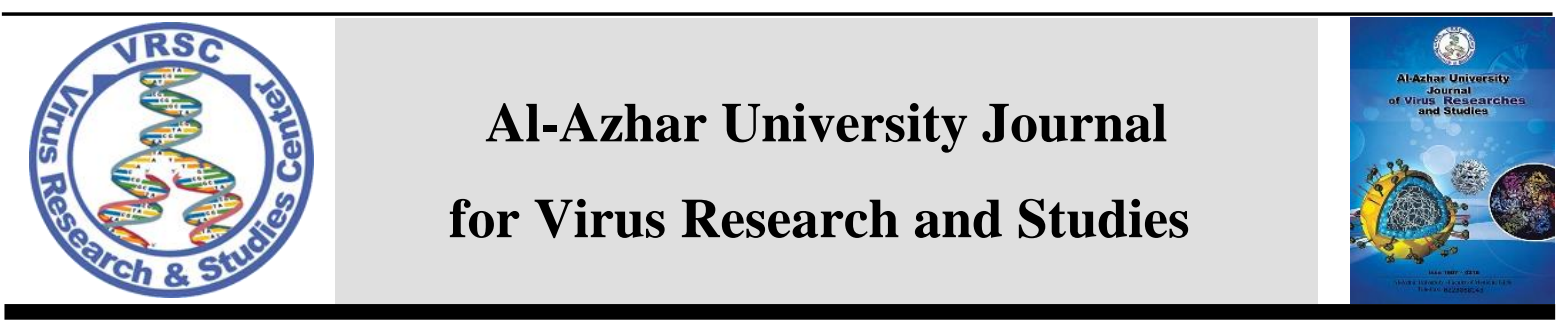

\title{
Automated Detection of Covid-19 Coronavirus Cases Using Deep Neural Networks with CT Images
}

\author{
Laila M Aboughazala, Kamel. K. Mohammed*
}

Center for Virus Research and Studies, Al-Azhar University, Cairo, Egypt

*E-mail: vrsc@azhar.edu.eg

\begin{abstract}
Early detection of COVID-19 based on chest CT will enable timely treatment of patients and help control the spread of the disease. With rapid spreading of COVID-19 in many countries, however, CT volumes of suspicious patients are increasing at a speed much faster than the availability of human experts. Here, we propose an artificial intelligence (AI) system for fast COVID-19 diagnosis with an accuracy comparable to experienced radiologists. A large dataset was constructed by collecting $746 \mathrm{CT}$ volumes of 359 patients with confirmed COVID-19 and 387 negative cases from publicly available chest CT datasets. In this paper, we propose a deep learning architecture to detect Covid-19 Coronavirus in CT Images. This architecture contains one network to classify images as either Covid-19 or Non-Covid-19. The experiment results evaluated by three parameters including accuracy, sensitivity, and specificity. For the ResNet50 deep learning, these outcomes refer to the maximum sensitivity being $91.69 \%$ by the training dataset for the ResNet-50. ResNet-50 can be considered as a high sensitivity model to characterize and diagnose Covid-19 Coronavirus and can be used as an adjuvant tool in radiology departments.
\end{abstract}

Keywords: COVID-19, CT images, Deep learning, ResNet-50 architecture.

\section{Introduction}

Actually, COVID-19 has had a major effect on industrialized and growing countries' economic and social systems [1,2]. In Wuhan, China, in December 2019, the initial case of COVID-19 illness was identified [3]. However, we also have to overcome the recent pandemic position $[4,5]$. By producing a volatile healthcare crisis infinitely more damaging than the Second World War, this disastrous impact has devastated human experience. COVID19 's clear communicable characteristics make the situation in the world a grave pandemic. A number of countries such as the U.S.A [6], Brazil [7], China [8] and
Italy [9] are seriously affected by this virus. Numerous study topics are therefore constructing policies, vaccines and innovative approaches to addressing this pandemic scenario [10]. Many scientists in the field of medicine create drugs to avoid virus prefoliation on the one side [11, 12]. Firstly, new ways of helping infected individuals must be developed [13-15]. Secondly, the development of health policies to avoid the spread of the virus by infected patients is also crucial $[16,17]$. In the development of new methods to support virus diagnostic $[18,19]$ computer science researchers have a critical role in this. 
utilized a relatively recent methodology, $\mathrm{CNN}$, where the learning methodology is developed to classify persons with COVID19 with a Resnet50. We follow the ResNet50 architecture as it has proven to be very successful for different medical imaging applications. Utilizing a collection of various technologies, many inventions have been made, for example the creation of mobile applications to control and follow interactions between persons [20,21]. Artificial intelligence (AI) is moreover essential in this area and in developing diagnostic support solutions [22]. Automated COVID-19 diagnostic systems were developed using AI methods [23-25]. Such approaches could never substitute human therapy. However, they could be an essential solution to the war against the virus. AI is generally utilized in medicine. Despite the ethical issue in the current pandemic scenario about the application of AI to patient data, these approaches should be utilized to assist medical personnel [2729]. Radiological images for the detection of COVID-19 cases utilizing typical deep learning methods were frequently utilized recently. Mohamed Loey et al. [30] introduced a computer-aided detection method based on deep learning that was able to detect COVID and Non-COVID from CT images. Techniques were used based on AlexNet, VGG16, VGG19, GoogleNet, and ResNet50. The CNN based pre-trained models are trained on 742 chest CT scans into two binary classes, i.e., COVID and Non-COVID. The highest accuracy of $82.91 \%$ is achieved with the ResNet50 pre-trained CNN model. The main objective of this study is to provide accurate measures for the identification of CT-image patients with COVID-19 via CNN.

\section{MATERIALS AND METHODS}

We used a publicly accessible dataset [31] [32] that includes Covid-19 or Non-Covid19. The dataset contains 746 CT images. The CT datasets images divided into 359 patients with confirmed COVID-19 and
387 non-COVID-19 CT images. Figure 1 represents certain sample images marked as normal by an expert reader. Figure 2 represents certain sample images marked as COVID-19 cases by an expert reader.

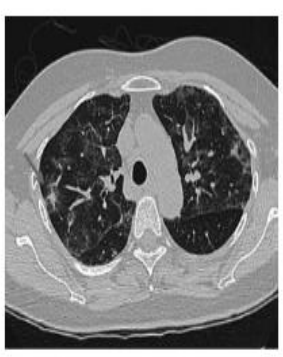

(a)

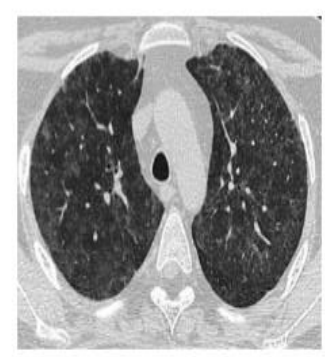

(b)
Figure 1. CT scans (a) for coronavirus and (b) for non-coronavirus images.

\subsection{Covid-19 Coronavirus Detection}

\section{Architecture}

The transfer learning-based CNN model approved for the classification of images as normal or COVID-19 is described in this portion. At first, in conjunction with the used architecture, we re-sample all images to $224 \times 224$. We used well-established networks such as ResNet-50 for transition learning purposes, as described above. The use of these well-established networks allows us to maintain a riches of knowledge for classifying various artefacts from previous training. Both weights and layers are maintained awaiting the final fully linked layer. Based on our data we will update ResNet-50. Since 1000 classes are trained in ResNet-50 million photos. In our case we have 746 images with 2 classes. With our own completely linked and softmax our last three layers are replaced. Particularly for image-based classification issues, these approaches have proven to be highly effective. The block diagram of the transfer learning method applied in this paper is presented in Figure 3. Crossvalidation is a model assessment technique used to evaluate a deep learning algorithm's performance in making predictions on new datasets that it has not been trained on. This is done by partitioning the known dataset, using a 
subset to train the algorithm and the remaining data for testing. Each round of cross-validation involves randomly partitioning the original dataset into a training set and a testing set. The training set is then used to train a supervised learning algorithm and the testing set is used to evaluate its performance. This process is repeated several times and the average cross-validation error is used as a performance indicator. We split the dataset into 10-folds for analysis i.e. 10 different algorithms would be trained using different set of images from the dataset. The 10 different algorithms contain set of training images and set of testing images from the dataset. This type of validation study would provide us a better estimate of our performance in comparison to typical holdout validation method.

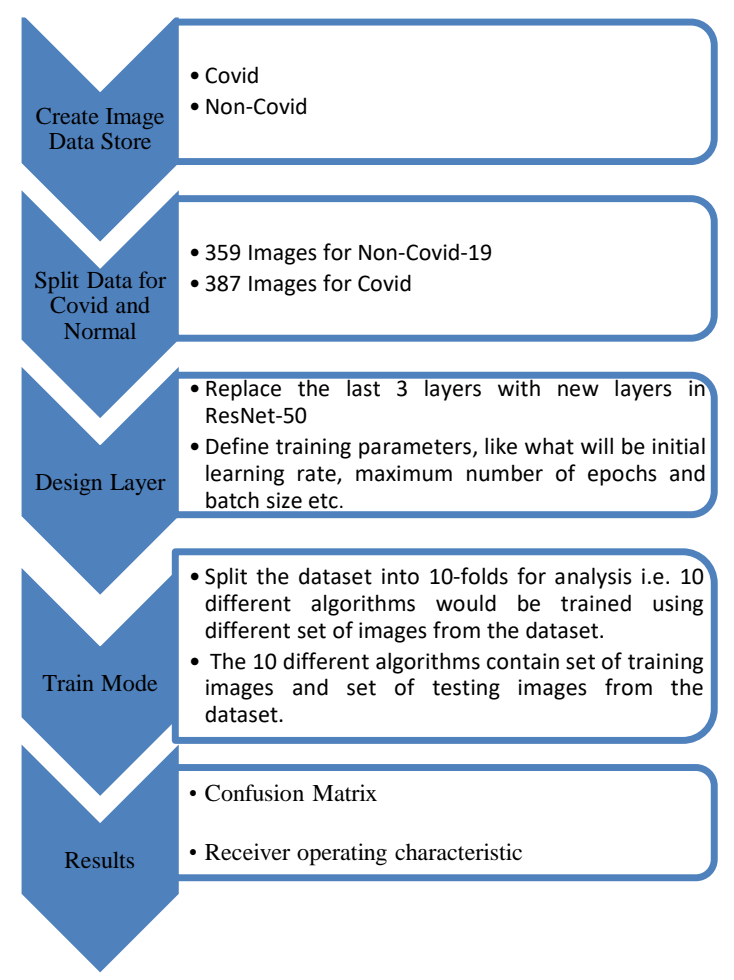

Figure 2. Block diagram of our algorithm.

\section{Experimental Results}

The results achieved for the method mentioned in sections 2 are discussed in this partition. The COVID-19 identification is determined on the basis of the datasets that composed of $746 \mathrm{CT}$ images and the data divided into $359 \mathrm{CT}$ images of patients with confirmed COVID-19 and 387 CT images for Non-COVID-19 CT images. First, the ResNet-50 model is trained to detect two classes: COVID-19 and NonCOVID-19 CT images categories. The performance of the proposed model is evaluated using the 10-fold crossvalidation procedure for the binary classification problem. Ninety per cent of CT images are used for training and ten per cent for testing. The experiments are repeated ten times. We have trained ResNet-50 for 30 epochs. Fig. 8 the training of the binary class classification graph is shown for the Fold-1. The overlapped as well as each separate confusion matrix (CM) are shown in Fig. 3, Fig. 4, Fig. 5, Fig. 6, and Fig. 7. The overlapped CM is created using the sum of CMs obtained in all folds. Thus, it is aimed to obtain an idea about the general perforations of the model. The ResNet-50 model achieved an average classification accuracy of $91.69 \%$ to classify COVID-19 CT images and NonCOVID-19 CT images. Sensitivity, specificity, precision, F1-score, and accuracy values are shown in Table 1 for the detailed analysis of the model for the two-class problem. It can be noted from the overlapped confusion matrix of the binaryclass classification task that the deep learning model classified COVID-19. The obtained sensitivity, specificity, and accuracy values are $97.14 \%, 95 \%$, and $91.69 \%$, respectively. It can be noted from Table 3 that the proposed model has achieved an average accuracy of $91.69 \%$ in detecting COVID-19 and the obtained average sensitivity, specificity, and F1score values of $97.14 \%, 95 \%$, and $91.69 \%$, respectively. In the COVID-19 epidemic, radiological imaging plays an important role in addition to the diagnostic tests performed for the early diagnosis, treatment, and isolation stages of the disease. CT images can detect a few characteristic findings in the lung 
associated with COVID-19. Deep learning models are sensitive in detecting COVID19 lung involvement and hence the diagnostic accuracy rate is high. During the evaluation of the model, CT images of COVID-19 patients confirmed positive by the PCR Test are used. The model can easily detect GGO, consolidation areas, and nodular opacities, which are the pathognomic findings of patients for COVID-19 on CT images. In COVID-19, bilateral, lower lobe, and peripheral involvement is observed, and the proposed model can detect the localization of the lesion. These models are particularly important in identifying early stages of COVID-19 patients. Early diagnosis of the disease is important to provide immediate treatment and to prevent disease transmission. The models can also play an indispensable role in patients lacking early symptoms. The clinical and radiological images of later-stage patients are well established, and it is easier to detect the findings by experts. The role of deep learning models is more prominent in screening and diagnosis when the infection is at its early stages time can be significantly reduced, and it will alleviate clinician workload.

\section{Conclusion}

In order to get an early detection of COVID-19 from CT images with the assistance of a computer-aided automated method, the contribution of this research is explained. The epidemic status scheme requires the correct $\mathrm{CT}$ images reader for COVID-19 via deep learning algorithms to minimize the prevalence of the virus, especially in low-income countries. Moreover, the usefulness of a system is supported by the vision of CT images of COVID-19. The satisfactory results were declared by the CNN classifier with high accuracy of $91.69 \%$. Lastly, this type of architecture would be highly essential for COVID-19 detection and its diagnosis and would enhance the workflow of radiologists.

Table 1: Sensitivity, specificity, precision, F1-score, and accuracy values for Normal and COVID-19 classes of the proposed model.

\begin{tabular}{|c|c|c|c|c|c|}
\hline Folds & Sensitivity & Specificity & Precision & F1-score & Accuracy \\
\hline 1 & 97.14 & 95 & 94.44 & 95.77 & 96 \\
\hline 2 & 94.29 & 95 & 94.29 & 94.29 & 94.67 \\
\hline 3 & 82.86 & 95 & 93.55 & 87.88 & 89.33 \\
\hline 4 & 97.14 & 87.50 & $\mathbf{8 7 . 1 8}$ & 91.89 & 92 \\
\hline 5 & 88.57 & 95 & 93.94 & 91.18 & 92 \\
\hline 6 & 91.43 & 90 & $\mathbf{8 8 . 8 9}$ & 90.14 & 90.67 \\
\hline 7 & 97.14 & $\mathbf{8 7 . 1 8}$ & $\mathbf{8 7 . 1 8}$ & $\mathbf{9 1 . 8 9}$ & 91.89 \\
\hline 8 & 97.14 & 87.18 & $\mathbf{8 7 . 1 8}$ & $\mathbf{9 1 . 8 9}$ & 91.89 \\
\hline 9 & 91.43 & 87.18 & $\mathbf{8 6 . 4 9}$ & $\mathbf{8 8 . 8 9}$ & $\mathbf{8 9 . 1 9}$ \\
\hline 10 & $\mathbf{8 8 . 2 4}$ & 90 & $\mathbf{8 8 . 2 4}$ & $\mathbf{8 8 . 2 4}$ & $\mathbf{8 9 . 1 9}$ \\
\hline Overlapped & 92.55 & 90.93 & $\mathbf{8 9 . 9 7}$ & 91.24 & 91.69 \\
\hline
\end{tabular}




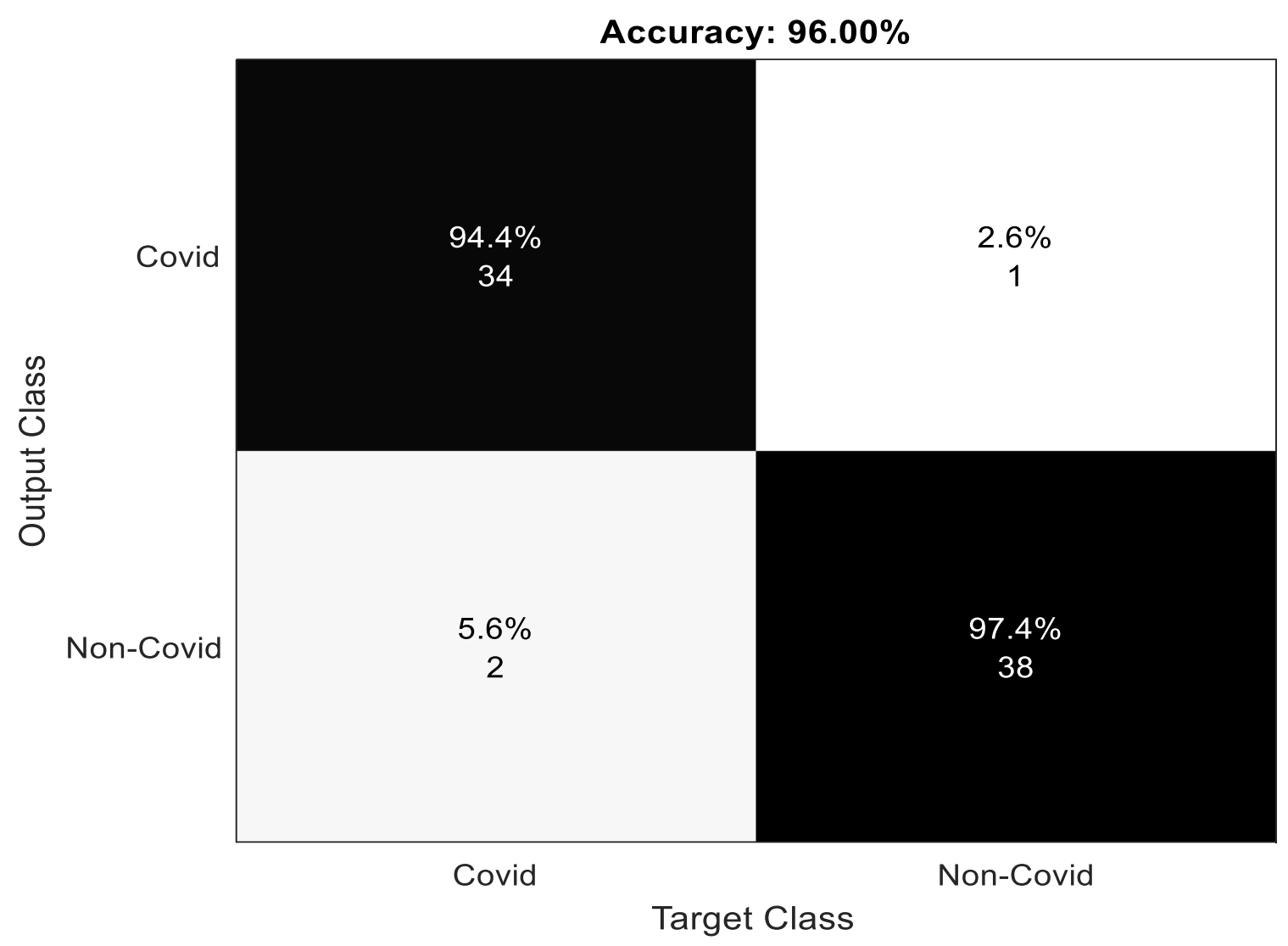

(a)

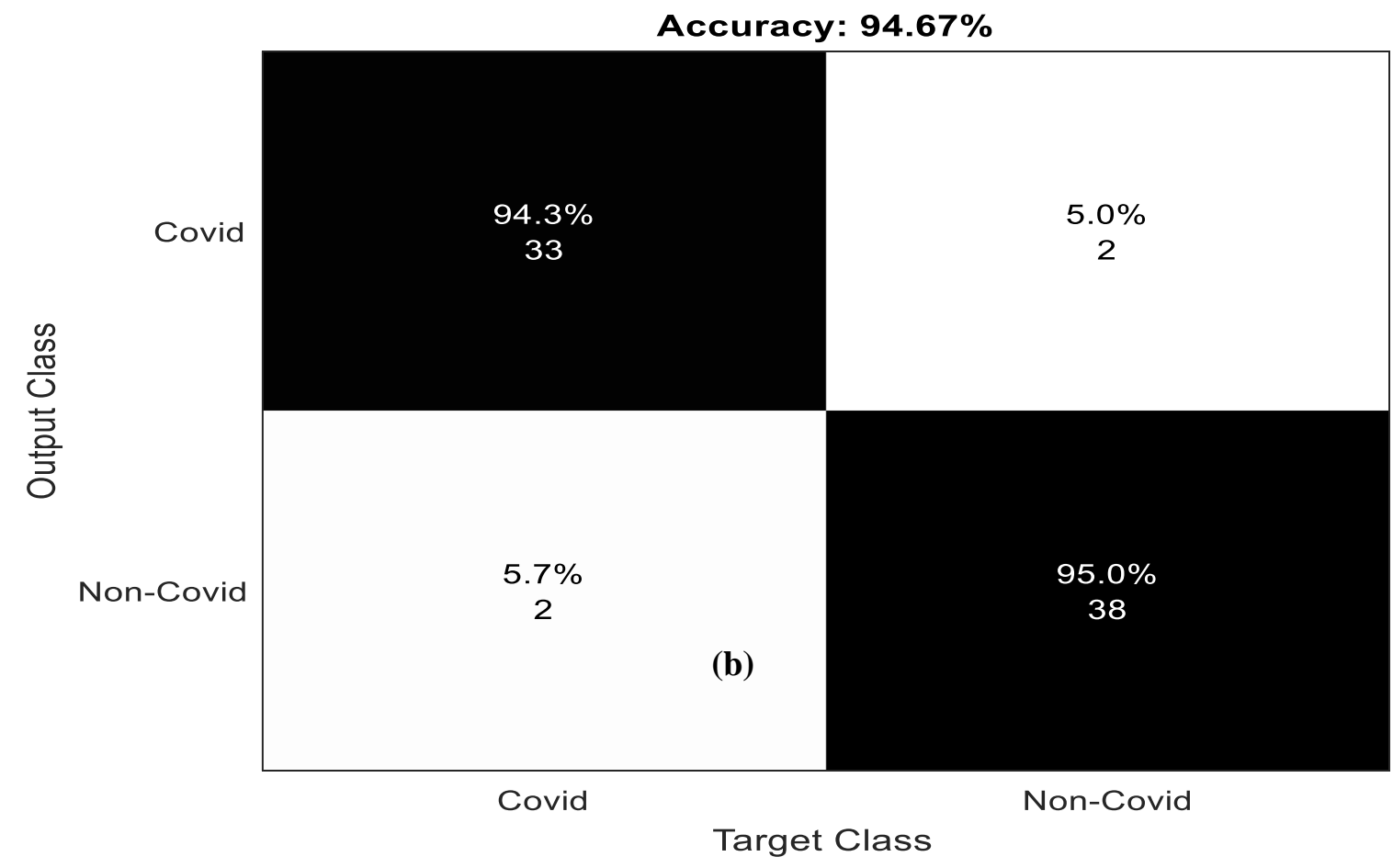

Fig. 3. The two-folds confusion matrix (CM) results of the binary classification task: (a) Fold-1 CM and (b) Fold-2 CM. 


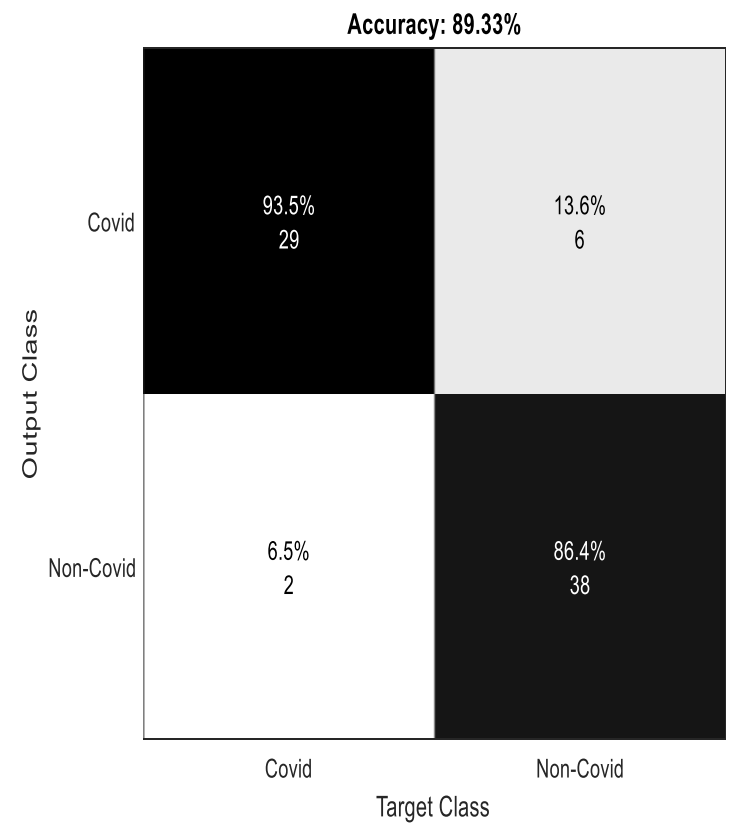

(a)

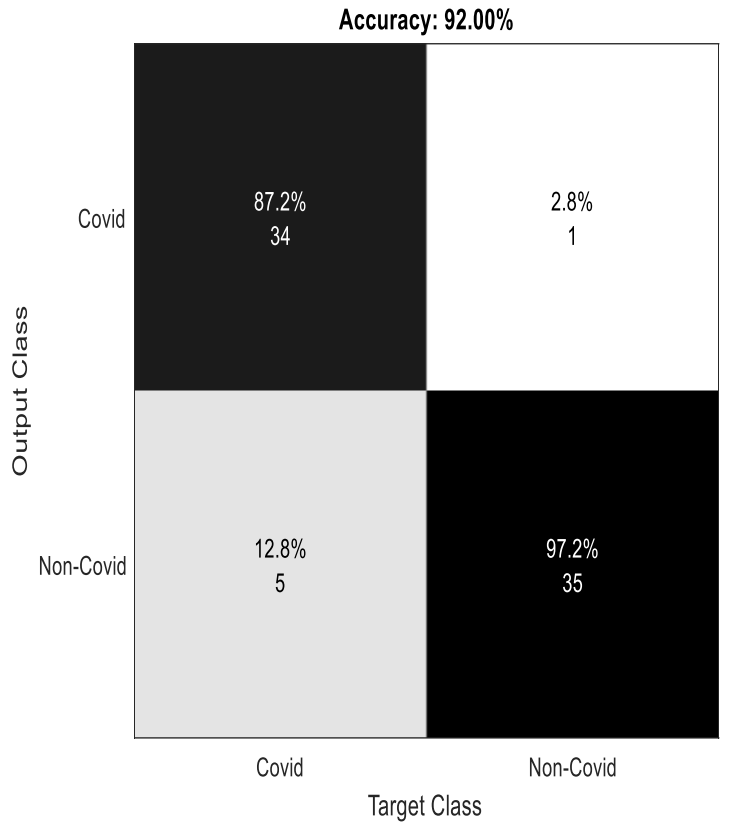

(b)

Fig. 4. The two-folds confusion matrix (CM) results of the binary classification task: (a) Fold-3 CM and (b) Fold-4 CM.

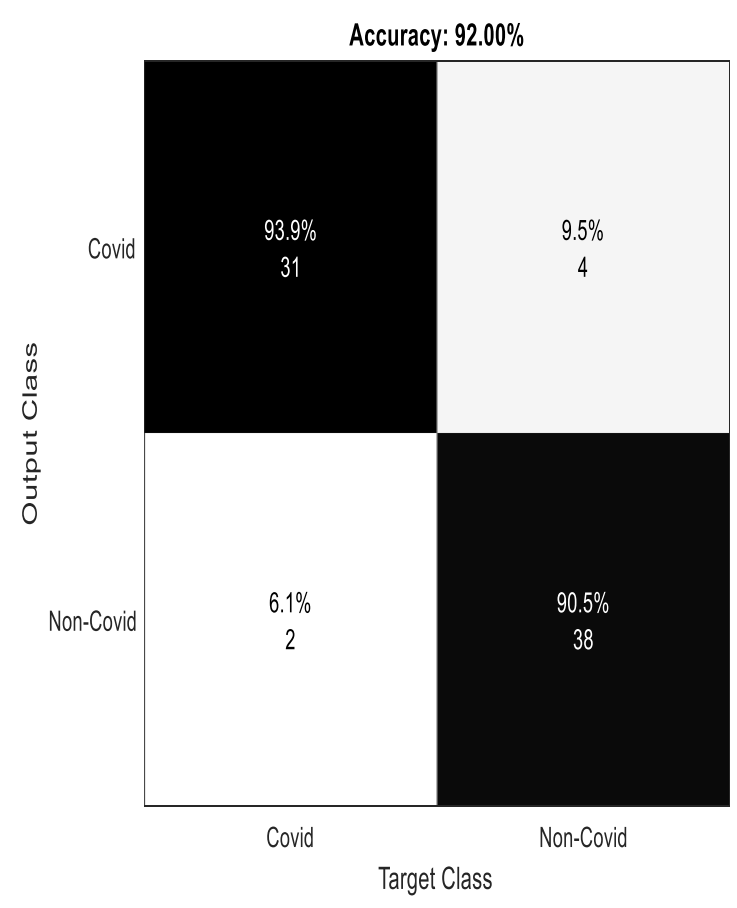

(a)

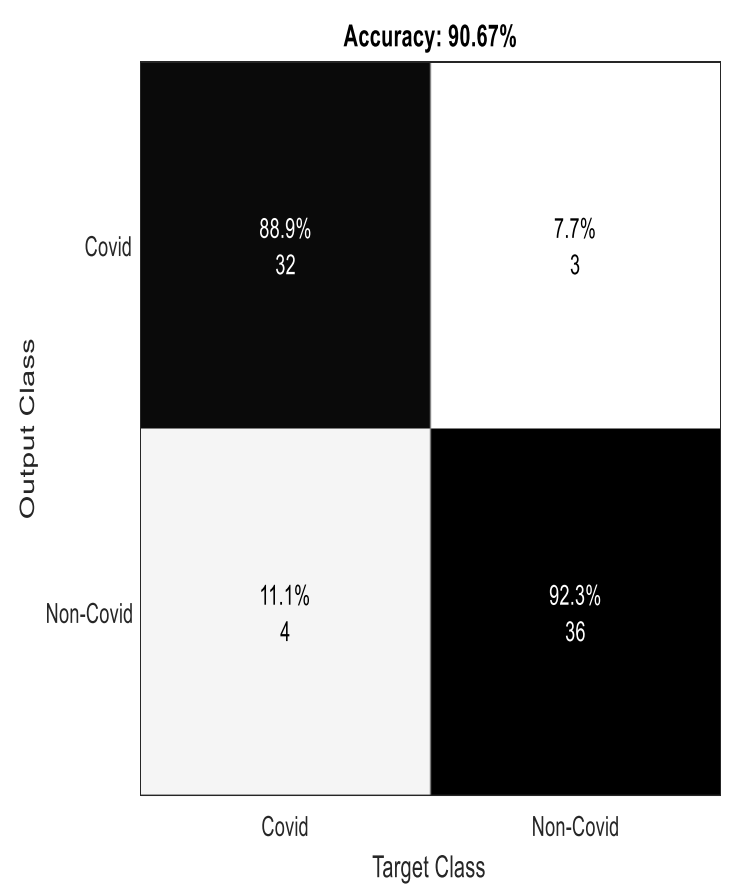

(b)

Fig. 5. The two-folds confusion matrix (CM) results of the binary classification task: (a) Fold-5 CM and (b) Fold-6 CM. 


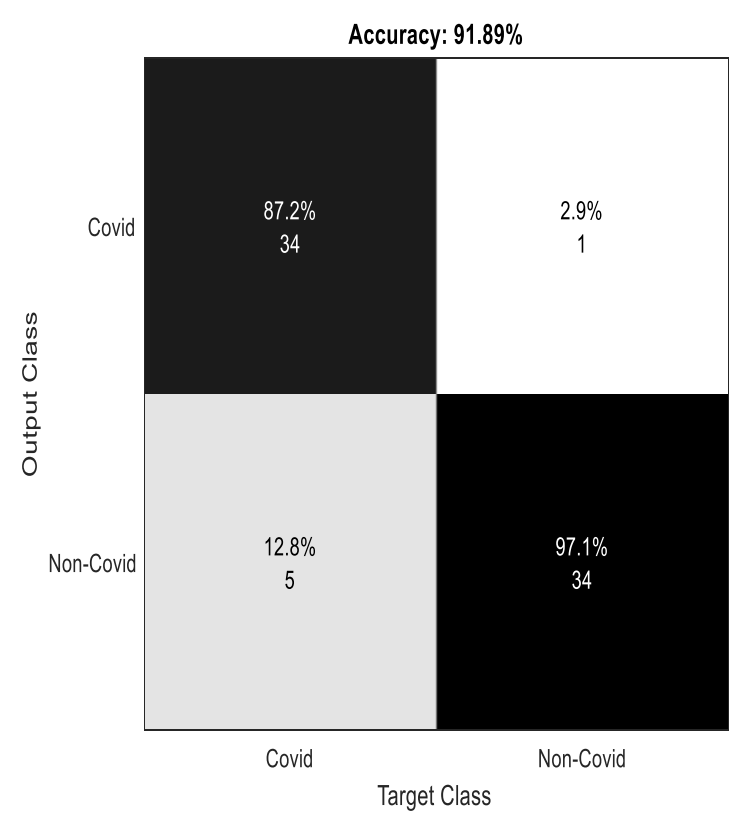

(a)

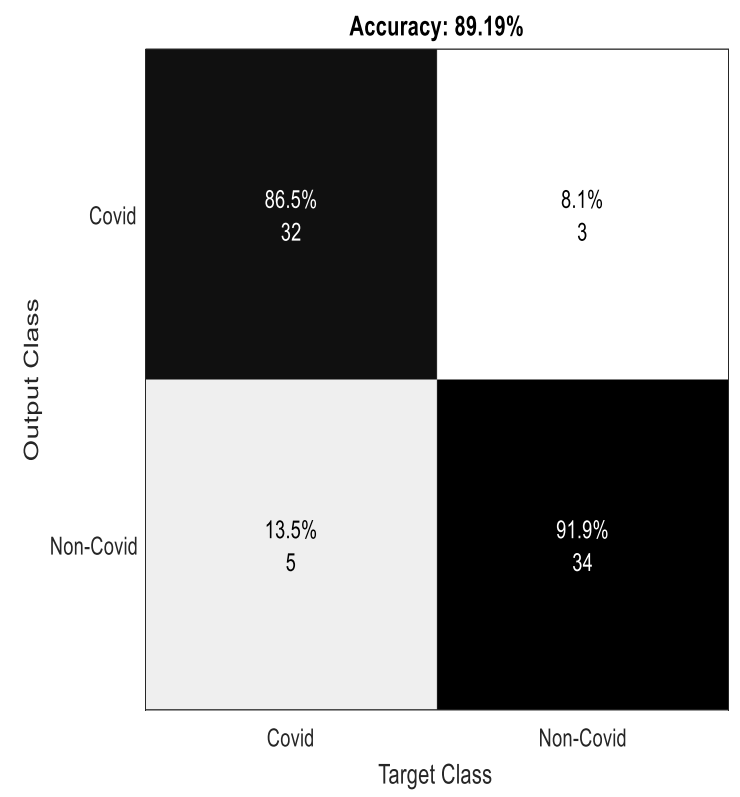

(b)

Fig. 6. The two-folds confusion matrix (CM) results of the binary classification task: (a) Fold-7,8 CM and (b) Fold-9 CM.

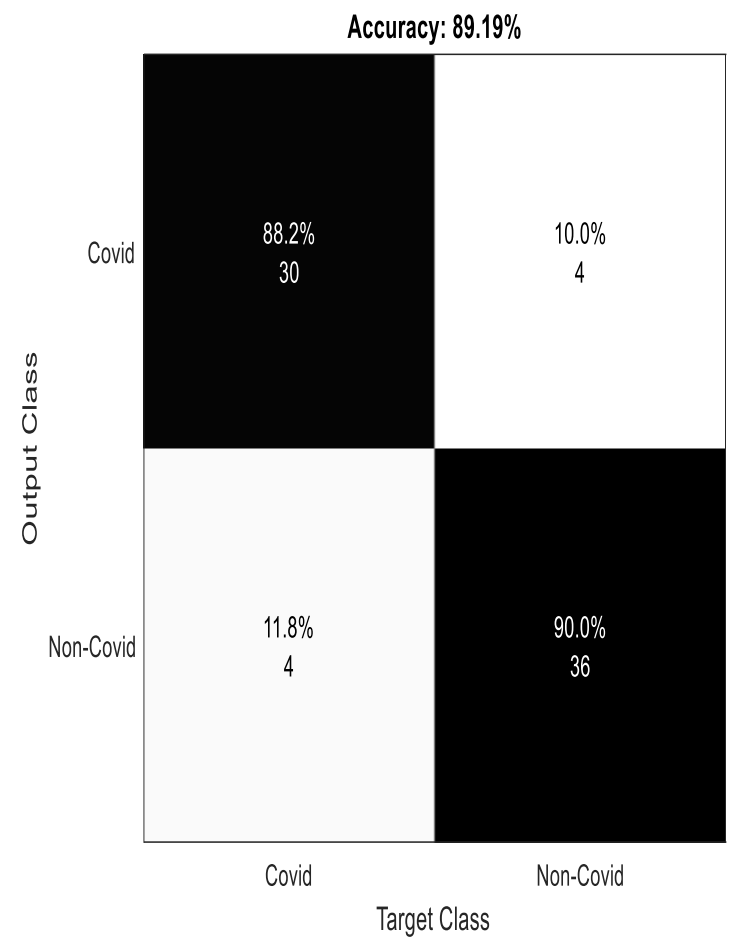

(a)

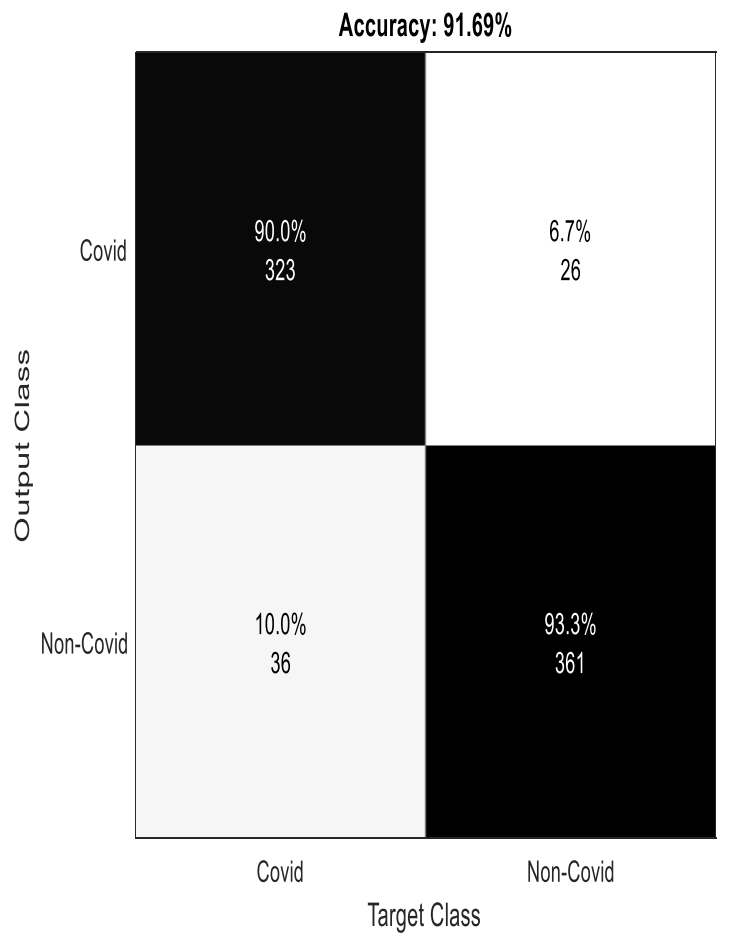

(b)

Fig. 7. The two-folds confusion matrix (CM) results of the binary classification task: (a) Fold-10 CM and (b) overlapped confusion matrix. 


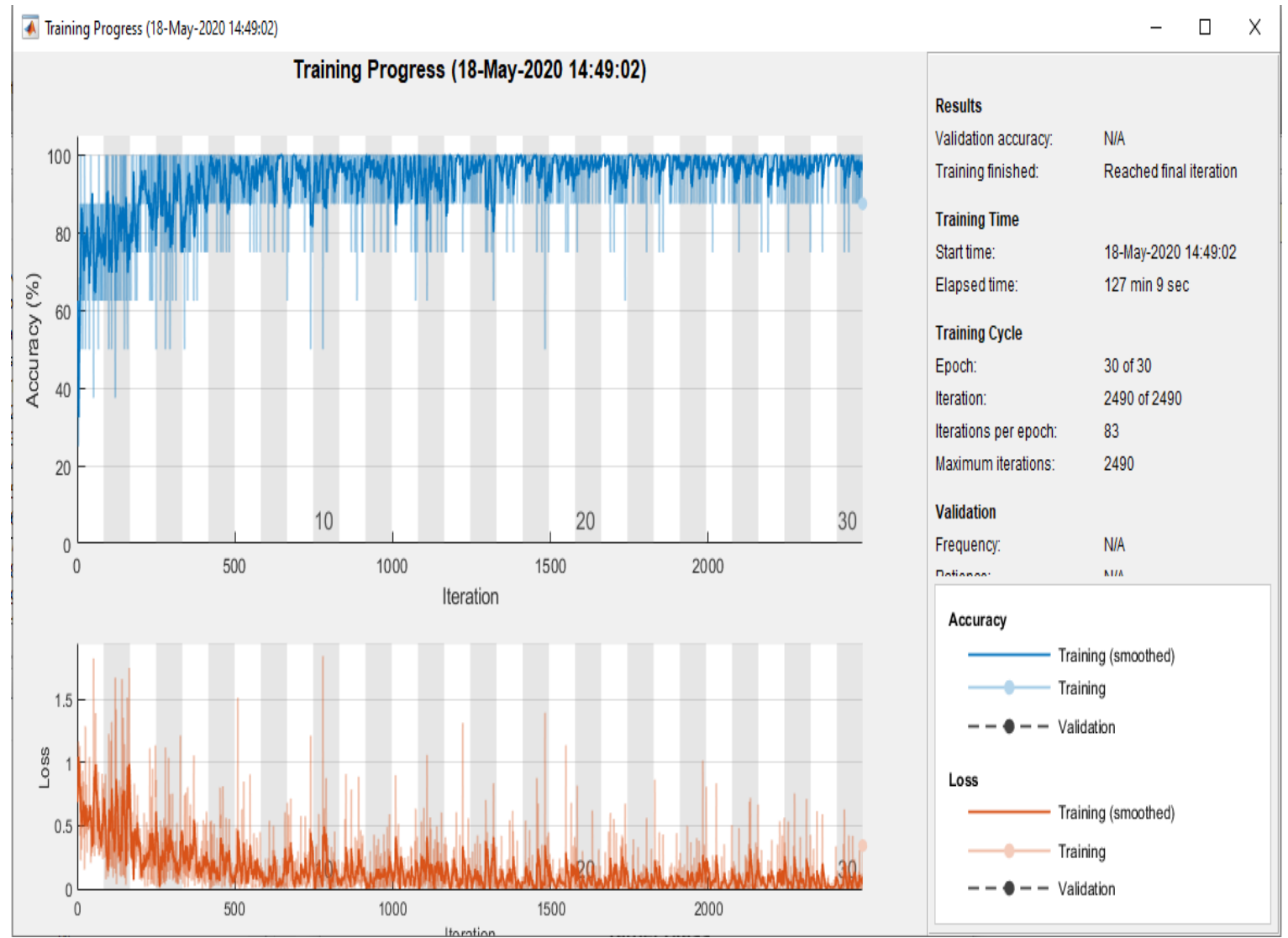

Fig 8: Training progress for ResNet-50 architecture on the dataset. 


\section{References}

1. C. Lodigiani, G. Iapichino, L. Carenzo, M. Cecconi, P. Ferrazzi, T. Sebastian, N. Kucher, J.-D. Studt, C. Sacco, B. Alexia, M.T. Sandri, S. Barco, Venous and arterial thromboembolic complications in COVID-19 patients admitted to an academic hospital in Milan, Italy, Thrombosis Res. 191 (2020) 9-14.

2. C. Huang, Y. Wang, X. Li, L. Ren, J. Zhao, Y. Hu, L. Zhang, G. Fan, J. $\mathrm{Xu}, \mathrm{X} . \mathrm{Gu}, \mathrm{Z}$. Cheng, T. Yu, J. Xia, Y. Wei, W. Wu, X. Xie, W. Yin, H. Li, M. Liu, Y. Xiao, H. Gao, L. Guo, J. Xie, G. Wang, R. Jiang, Z. Gao, Q. Jin, J. Wang, B. Cao, Clinical features of patients infected with 2019 novel coronavirus in Wuhan, China, Lancet 395 (2020) 497-506.

3. Y.-Y. Zheng, Y.-T. Ma, J.-Y. Zhang, X. Xie, COVID-19 and the cardiovascular system, Nat. Rev. Cardiol. 17 (2020) 259-260.

4. Imperial College COVID-19 Response Team, S. Flaxman, S. Mishra, A. Gandy, H.J.T. Unwin, T.A. Mellan, H. Coupland, C. Whittaker, H. Zhu, T. Berah, J.W. Eaton, M. Monod, A.C. Ghani, C.A. Donnelly, S. Riley, M.A.C. Vollmer, N.M. Ferguson, L.C. Okell, S. Bhatt, Estimating the effects of non-pharmaceutical interventions on COVID-19 in Europe, Nature 584 (2020) 257261.

5. F.-X. Lescure, L. Bouadma, D. Nguyen, M. Parisey, P.-H. Wicky, S. Behillil, A. Gaymard, M. Bouscambert-Duchamp, F. Donati, Q. Le Hingrat, V. Enouf, N. Houhou-Fidouh, M. Valette, A.
Mailles, J.-C. Lucet, F. Mentre, X. Duval, D. Descamps, D. Malvy, J.F. Timsit, B. Lina, S. van-der Werf, Y. Yazdanpanah, Clinical and virological data of the first cases of COVID-19 in Europe: A case series, Lancet Infectious Dis. 20 (2020) 697-706.

6. A.P. Kansagra, M.S. Goyal, S. Hamilton, G.W. Albers, Collateral effect of Covid-19 on stroke evaluation in the United States, New Engl. J. Med. 383 (2020) 400 401.

7. A.J. Rodriguez-Morales, V. Gallego, J.P. Escalera-Antezana, C.A. Méndez, L.I. Zambrano, C. Franco-Paredes, J.A. Suárez, H.D. Rodriguez-Enciso, G.J. BalbinRamon, E. Savio-Larriera, A. Risquez, S. Cimerman, COVID-19 in Latin America: The implications of the first confirmed case in Brazil, Travel Med. Infectious Dis. 35 (2020).

8. D. Roy, S. Tripathy, S.K. Kar, N. Sharma, S.K. Verma, V. Kaushal, Study of knowledge, attitude, anxiety \& perceived mental healthcare need in Indian population during COVID-19 pandemic, Asian J. Psychiatry 51 (2020)

9. A. Remuzzi, G. Remuzzi, COVID19 and Italy: What Next? Lancet 395 (2020) 1225-1228.

10. H. Harapan, N. Itoh, A. Yufika, W. Winardi, S. Keam, H. Te, D. Megawati, Z. Hayati, A.L. Wagner, M. Mudatsir, Coronavirus disease 2019 (COVID-19): A literature review, J. Infection Public Health 13 (2020) 667-673. 
11. X. Cao, COVID-19: Immunopathology And its implications for therapy, Nat. Rev. Immunol. 20 (2020) 269-270.

12. P. Mehta, D.F. McAuley, M. Brown, E. Sanchez, R.S. Tattersall, J.J. Manson, COVID-19: consider cytokine storm syndromes and immunosuppression, Lancet 395 (2020) 1033-1034.

13. S. Al Kasab, E. Almallouhi, C.A. Holmstedt, Optimizing the Use of Teleneurology During the COVID19.

14. A. Doshi, Y. Platt, J.R. Dressen, B.K. Mathews, J.C. Siy, Keep calm and $\log$ on: Telemedicine for COVID-19 pandemic response, 2020.

15. S. Omboni, Telemedicine During The COVID-19 in Italy: A Missed Opportunity?

16. A. Tashiro, R. Shaw, COVID-19 pandemic response in Japan: What is behind the initial flattening of the curve? Sustainability 12 (2020).

17. D. Lee, K. Heo, Y. Seo, COVID-19 in South Korea: Lessons for developing countries, World Dev. 135 (2020).

18. M. Ostaszewski, A. Mazein, M.E. Gillespie, I. Kuperstein, A. Niarakis, H. Hermjakob, A.R. Pico, E.L. Willighagen, C.T. Evelo, J. Hasenauer, F. Schreiber, A. Dräger, E. Demir, O. Wolkenhauer, L.I. Furlong, E. Barillot, J. Dopazo, A. Orta-Resendiz, F. Messina, A. Valencia, A. Funahashi, H. Kitano, C. Auffray, R. Balling, R. Schneider, COVID-19 disease map, building a computational repository of SARS-CoV-2 virus-host interaction mechanisms, Sci Data. (2020).

19. N. Muralidharan, R. Sakthivel, D. Velmurugan, M.M. Gromiha, Computational studies of drug repurposing and synergism of lopinavir, oseltamivir and ritonavir binding with SARS-CoV-2 protease against COVID-19, J. Biomol. Struct. Dyn. (2020) 1-6.

20. Z. Li, J. Ge, M. Yang, J. Feng, M. Qiao, R. Jiang, J. Bi, G. Zhan, X. $\mathrm{Xu}, \mathrm{L}$. Wang, Q. Zhou, C. Zhou, Y. Pan, S. Liu, H. Zhang, J. Yang, B. Zhu, Y. Hu, K. Hashimoto, Y. Jia, H. Wang, R. Wang, C. Liu, C. Yang, Vicarious traumatization in the general public, members, and non-members of medical teams aiding in COVID-19 control, Brain, Behav. Immunity 88 (2020) 916919.

21. D.A. Drew, L.H. Nguyen, C.J. Steves, C. Menni, M. Freydin, T. Varsavsky, C.H. Sudre, M.J. Cardoso, S. Ourselin, J. Wolf, T.D. Spector, A.T. Chan, COPE Consortium§: Rapid implementation of mobile technology for Realtime epidemiology of COVID-19, Science. 368 (2020) 1362-1367.

22. F. Shi, J. Wang, J. Shi, Z. Wu, Q. Wang, Z. Tang, K. He, Y. Shi, D. Shen, Review of artificial intelligence techniques in imaging data acquisition, segmentation and diagnosis for COVID-19, IEEE Rev. Biomed. Eng. 12020.

23. A. Alimadadi, S. Aryal, I. Manandhar, P.B. Munroe, B. Joe, $\mathrm{X}$. Cheng, Artificial intelligence and machine learning to fight COVID-19, Physiol. Genomics 52 (2020) 200-202. 
24. A.S.R. Srinivasa Rao, J.A. Vazquez, Identification of COVID19 can be quicker through artificial intelligence framework using a mobile phone-based survey when cities and towns are under quarantine, Infect. Control Hosp. Epidemiol. 41 (2020) 826-830.

25. R. Vaishya, M. Javaid, I.H. Khan, A. Haleem, Artificial intelligence (AI) applications for COVID-19 pandemic, Diabetes Metab. Syndr: Clin. Res. Rev. 14 (2020) 337-339.

26. E.J. Topol, High-performance medicine: the convergence of human and artificial intelligence, Nat. Med. 25 (2019) 44-56.

27. I.V. Buzaev, V.V. Plechev, I.E. Nikolaeva, R.M. Galimova, Artificial intelligence: Neural network model as the multidisciplinary team member in clinical decision support to avoid medical mistakes, Chronic Dis. Transl. Med. 2 (2016) 166-172.

28. F.H.D. Araújo, A.M. Santana, P. de A. Santos Neto, using machine learning to support healthcare professionals in making preauthorisation decisions, Int. J. Med. Inform. 94 (2016) 1-7.

29. L. Luo, J. Li, C. Liu, W. Shen, using machine-learning methods to support health-care professionals in making admission decisions, Int. J. Health Plann. Mgmt. 34 (2019).

30. Mohamed Loey, Florentin Smarandache, and Nour Eldeen M. Khalifa. A deep transfer learning model with classical data augmentation and cgan to detect covid-19 from chest ct radiography digital images. J Neural Computing and Applications, 2020.
31. Zhao, J., Zhang, Y., He, X., Xie, P. (2020). COVID-CT-dataset: A CT scan dataset about COVID-19. arXiv preprint arXiv:2003.13865.

32. Heba M. Afify, Ashraf Darwish, Kamel K. Mohammed, Aboul Ella Hassanien. "An Automated CAD System of CT Chest Images for COVID-19 Based on Genetic Algorithm and K-Nearest Neighbor Classifier", Ingénierie des systèmes d information, 2020 